\title{
The Relationship between MR Parameters and Biomechanical Quantities of Loaded Human Articular Cartilage in Osteoarthritis: An In-Vitro Study
}

\author{
V. Juráś ${ }^{1,2}$, P. Szomolányi ${ }^{1,2}$, S. Gäbler ${ }^{3}$, I. Frollo $^{2}$, S. Trattnig $^{1}$ \\ ${ }^{1}$ MR Center, Highfield MR, Department of Radiology, Medical University of Vienna, Lazarettgasse 14, 1090 Vienna, Austria, \\ ${ }^{2}$ Department of Imaging Methods, Institute of Measurement Science, Slovak Academy of Sciences, Dúbravska cesta 9, \\ 84104 Bratislava, Slovakia, \\ ${ }^{3} \mathrm{TU}$ Wien, Institute of Materials Science and Technology, Vienna, Austria \\ Email: umervjur@savba.sk
}

\begin{abstract}
The aim of this study was to assess the changes in MRI parameters during applied load directly in MR scanner and correlate these changes with biomechanical parameters of human articular cartilage. Cartilage explants from patients who underwent total knee replacement were examined in the micro-imaging system in $3 T$ scanner. Respective MRI parameters $\left(T_{1}\right.$ without- and $T_{1}$ with contrast agent as a marker of proteoglycan content, $T_{2}$ as a marker of collagen network anisotropy and ADC as a measure of diffusivity) were calculated in pre- and during compression state. Subsequently, these parameters were compared to the biomechanical properties of articular cartilage, instantaneous modulus (I), equilibrium modulus (Eq) and time of tissue relaxation $(\tau)$. Significant load-induced changes of $T_{2}$ and ADC were recorded. High correlation between T1Gd and $I(r=0.6324)$, and between ADC and Eq $(r=-\mathbf{0 . 4 8 8 4})$ was found. Multi-parametric MRI may have great potential in analyzing static and dynamic biomechanical behavior of articular cartilage in early stages of osteoarthritis (OA).
\end{abstract}

Keywords: Articular cartilage, MRI, biomechanics, compression

\section{INTRODUCTION}

$\mathrm{O}$ NLY LIMITED information is currently available on the relationship between cartilage compression and signal changes in $\mathrm{T}_{1}, \mathrm{~T}_{2}$ and diffusion weighted images as a consequence of biochemical and biomechanical alterations during and after compression. In order to interpret medical images from MRI correctly, one should know several circumstances that precede the measurement itself. Cartilage compression can be considered as one of these factors. Cartilage tissue consists of several types of macromolecules that provide basic functions: to distribute the load within a joint and provide a smooth surface for articulation. Load distribution in the joint is influenced by cartilage anatomy and mechanical properties, the presence of menisci and ligaments, bone stiffness and anatomy, and loading direction and kinetics. MR imaging has become the method of choice in the evaluation of normal [1] and damaged [2] cartilage due to the improved soft tissue contrast and multi planar capability without radiation exposure. Several studies were performed in the field of mechanical testing of cartilage during compression [3]. Biochemical and biomechanical changes were observed: fluid-flow and internal deformation, intrinsic viscoelasticity, changes in the water content or catabolism and loss of proteoglycans [4]. Investigators attempted to use MRI to evaluate mechanical properties of cartilage after applying load. In some cases, an MR-compatible device was built for controlled loading of cartilage explants and intact joints [5]. In another study, the rate and degree of deformation were increased after trypsin degradation [6]. A recent in vivo study demonstrated the ability to measure changes in cartilage volume as a function of mechanical stress (i.e. exercise) [7]. These studies imply that MRI is a modality with sufficient sensitivity to evaluate load-induced changes in cartilage tissue.
The goal of this study was to evaluate common MRI parameters of human articular cartilage and their changes as a consequence of static compression by a special designed nonmagnetic device for indentation tests of cartilage tissue and correlate these with selected biomechanical parameters.

\section{SubJeCt \& Methods}

Experiments were performed on a Bruker 3T Medspec whole-body scanner (Bruker, Ettlingen, Germany). A BGA 12 micro-imaging gradient system capable of delivering a $200 \mathrm{mT} / \mathrm{m}$ gradient, and $35 \mathrm{~mm}$ inner diameter resonator was used (functional diagram is depicted in Fig.1). The test equipment for micro-imaging of cartilage under compression consists of a micro-gradient (Fig.2a), a coil holder (Fig. 2b) for fixing the coil, a custom built waterproof chamber for holding the sample (Fig.2c), a further plastic chamber (Fig.2f) and compression rod with a displacement scale (Fig.2e). The micro-gradient system is actively shielded and water-cooled with an aperture diameter of $25 \mathrm{~mm}$. In order to fasten the rig into the whole-body $3 \mathrm{~T}$ scanner two nylon screws fixed the rig to the appropriate holes in the patient table [8].

Cartilage samples were prepared from joints of 10 patients, who underwent total knee joint replacement. The samples were cuboid-shaped, with $10 \times 10 \times 6 \mathrm{~mm}$ in dimension. The study was performed on a Bruker 3T Medspec whole-body scanner (Bruker, Ettlingen, Germany) using BGA-12 microgradients (capable of delivering $200 \mathrm{mT} / \mathrm{m}$ ) with a special designed compression device built for this gradient system. The cartilage sample was compressed in a way that $15 \%$ of thickness decrease was accomplished.

$\mathrm{T}_{1}$ mapping was performed after filling the water-proof chamber of the compression device with a $2 \mathrm{mmol}$ solution of 


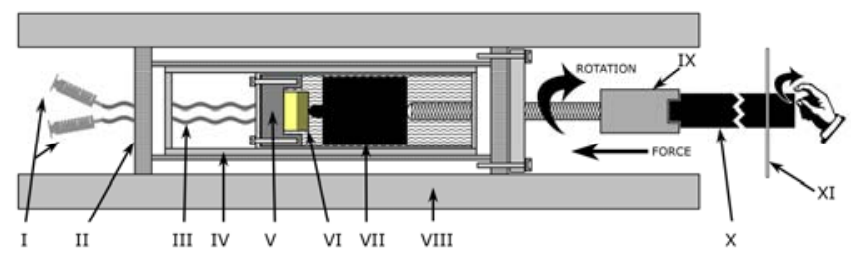

Fig.1 Function diagram of compression device: I. Syringes used for liquid content swapping; II. Coil holder; III. Hoses connected to waterproof chamber; IV. Coil with $35 \mathrm{~mm}$ inner diameter; V. Plastic chamber; VI. Cartilage sample - brighter area represents bone tissue, darker area represents cartilage tissue; VII. Compressive piston there are more types with different contact areas, the one depicted has contact area of $3 \mathrm{~mm}^{2}$; VIII. The body of the micro-gradient insert; IX. Screw that produces compressive force with direction represented by the large left- arrow; X. $90 \mathrm{~cm}$ rod allows to perform compression out of the magnet; XI. Scale for exact displacement of compressive piston.

gadopentetate dimeglumine (Gd-DTPA, Schering, Berlin, Germany) and soaking the cartilage sample in the solution for 24 hours. $T_{1}$ mapping was realized by spin echo pulse sequence with inversion recovery, TI times were set to 15,30 , 60, 160, 400 and $2000 \mathrm{~ms}$. Each pixel intensity of the individual images was then fitted to the function

$$
S(T I)=S_{0}\left(1-2 e^{-T I / T_{1}}\right)
$$

where $\mathrm{S}(\mathrm{TI})$ is for the intensity of the pixel at the inversion time TI.

For $\mathrm{T}_{2}$ mapping, multi-echo multi-slice spin echo sequence with TE times $15,30,45,60,75$ and 90 ms was used. Intensities of individual TE images were fitted to the function:

$$
S(T E)=S_{0} e^{-T E / T_{2}}+B
$$

where B means the baseline which is defined by hardware capabilities.

ADCs were calculated from data from pulsed gradient spin echo (PGSE) with 6 different b-values $(10.472,220$. 627, $452.8,724.5$ and 957.7). $b$-value is defined in terms of

$$
b=\gamma^{2} A^{2} \delta^{2}(\Delta-\delta / 3)
$$

where $\gamma$ is the gyromagnetic ratio of proton, $\mathrm{A}$ is the amplitude of gradient pulses, $\delta$ is their duration, and $\Delta$ is the time between ramps. Intensities of pixels from individual images were fitted to the function:

$$
S(b)=S_{0} e^{-b A D C}
$$

Other sequence parameters were set similarly for each parameter calculation: TR, $4000 \mathrm{~ms}$ (in case of IR, TR was incremented by $\mathrm{TI}$, i.e. $\mathrm{TR}_{\mathrm{IR}}=\mathrm{TR}+\mathrm{TI}$ ); matrix size, $128 \mathrm{x}$ 128; FOV, 30 x $30 \mathrm{~mm}$; bandwidth, $15 \mathrm{kHz}$. Time of measurement resulted from sequence settings as follows: $T_{1}, 6$ x $38 \mathrm{~min} ; \mathrm{T}_{2}, 39 \mathrm{~min}$; ADC, $38 \mathrm{~min}$.
Each of the parameters was calculated by fitting on pixel-bypixel basis to the appropriate function. Fitting routines were written in IDL (Interactive Data Language, Research Systems, Inc.) using mpcurvefit routine [9]. Region of interests (ROI) were set in appropriate place where the indenter was in contact with cartilage surface. Measurement setup and the specificity of the device allowed choosing one ROI only for pre- and post-compression state which maintained the accuracy of ROI selection through the individual maps. ROI was selected immediately near the indenter and evaluated at once for $T_{1}, T_{2}$, and ADC maps in a non-compressed and a compressed state. Since the micro-gradient insert allows data acquisition with high in-plane resolution $(234 \mu \mathrm{m})$, it was possible to conveniently select three different cartilage zones (superficial, transitional, and radial). The zones were selected as follows: the superficial zone was defined as the $16 \%$ of cartilage from the cartilage surface straight down to deeper tissue; the middle zone was defined as the zone $31 \%$ adjacent to the superficial zone; and the deep zone was defined as the remaining $53 \%$ of the cartilage down to the cartilage-bone interface. a

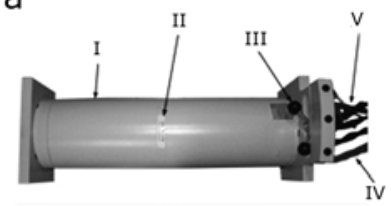

C
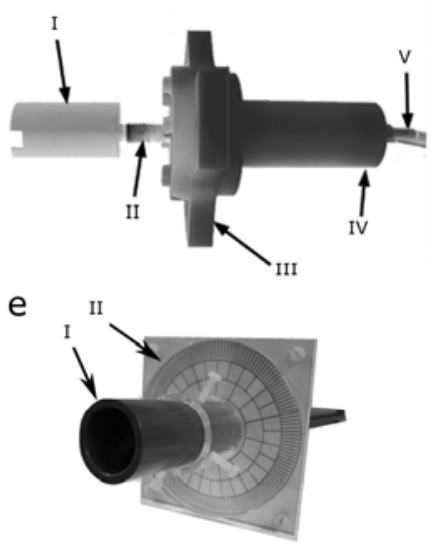

b

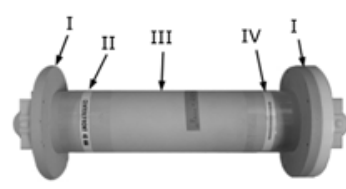

d

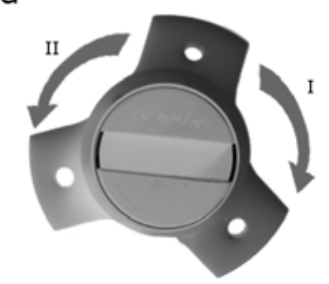

f

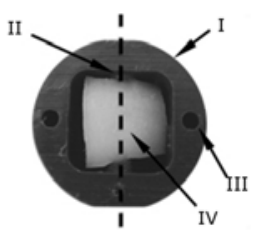

Fig.2 a: Micro-gradient system BGA 12: I - body of the device, II - center mark, III - top fixing screw, IV - hoses for water cooling, V - supply cables. b: Coil holder - top view: I - discs to fit this part into a micro-gradient insert, II - mark for correct direction, III - connecting cylinder, IV - mark for correct position in scanner. c: Sample holder - top view: I, II - screw for generating compression movement, III - milled disc for fixing holder to coil holder, IV - waterproof chamber with cartilage sample, V - slender hoses. d: Sample holder - left view: compression of cartilage is accomplished by rotating the screw in direction I, decompression in direction II. e: I - compression rod, II - scale (the smallest segment corresponds to $1 / 150 \mathrm{~mm})$. f: Sample holder: I - plastic body, II - screws for cartilage fixing, III - screw holes for holder fixing, IV - piece of the cartilage.

Regarding biomechanical parameters, Eq (equilibrium modulus), I (instantaneous modulus) and $\tau$ (time of tissue relaxation) were measured by indentation tests on a Zwick Z050 universal testing device with a $20 \mathrm{~N}$-load cell of $1 \mathrm{mN}$ 
resolution. To determine biomechanical parameters, indentation test suggested by Hayes was employed [10]. Moduli were calculated from the formula

$$
E=\frac{F(1-v)^{2}}{2 r_{a c t} s \kappa}
$$

where $\kappa$ is the dimensionless scale factor, $\mathrm{F}$ is the load in $\mathrm{N}, v$ is the Poisson ratio, $r_{a c t}$ is the actual indenter area in $\mathrm{mm}, \mathrm{G}$ is the shear modulus in $\mathrm{N} / \mathrm{mm}^{2}$ a $s$ is the depth of indenter penetration. Equilibrium modulus was calculated after $30 \mathrm{~min}$ of static loading. OA condition was determined by histological evaluation using hematoxylin-eosine staining. Biomechanical and MR parameters were compared using Pearson correlation coefficient and statistical significance was determined by the pair T-test ( $\mathrm{p}$-value lower than 0.05 was considered as statistical significant).

\section{RESULTS}

Table 1. Values of $T_{1}, T_{2}$ and ADC, comparison of pre- and during-compression states

\begin{tabular}{llll}
\hline & $\begin{array}{l}\mathrm{ADC} \\
{\left[10^{-3} \mathrm{~mm}^{2} / \mathrm{s}\right]}\end{array}$ & $\begin{array}{l}\mathrm{T}_{1} \\
{[\mathrm{~ms}]}\end{array}$ & $\begin{array}{l}\mathrm{T}_{2} \\
{[\mathrm{~ms}]}\end{array}$ \\
\hline $\begin{array}{l}\text { number of pixels in } \\
\text { ROI }\end{array}$ & 166 & 110 & 108 \\
$\begin{array}{l}\text { without } \\
\text { compression }\end{array}$ & $0.96 \pm 0.40$ & $180 \pm 30$ & $30 \pm 9$ \\
$\begin{array}{l}\text { with } \\
\text { compression }\end{array}$ & $0.85 \pm 0.39$ & $230 \pm 30$ & $27 \pm 8$ \\
change $(\%, \mathrm{p}$ value $)$ & $\begin{array}{l}11 \% \\
(<0.05)\end{array}$ & $\begin{array}{l}22 \% \\
(0.238)\end{array}$ & $\begin{array}{l}10 \% \\
(<0.05)\end{array}$ \\
\hline
\end{tabular}

Table 2. Pearson correlation coefficients between MR parameters during loading and biomechanical parameters measured by indentation tests

\begin{tabular}{|c|c|c|c|c|}
\hline & $\begin{array}{l}\mathrm{Eq} \\
{[\mathrm{MPa}]}\end{array}$ & $\begin{array}{l}\text { I } \\
{[\mathrm{MPa}]}\end{array}$ & $\mathrm{I} / \mathrm{Eq}$ & $\begin{array}{l}\tau \\
{[\mathrm{s}]} \\
\end{array}$ \\
\hline $\mathrm{T} 1 \mathrm{c} / \mathrm{T} 1 \mathrm{w}$ & -0.1884 & 0.6324 & -0.5275 & 0.6092 \\
\hline $\mathrm{T} 2 \mathrm{c} / \mathrm{T} 1 \mathrm{w}$ & 0.3620 & 0.1118 & -0.0860 & 0.0257 \\
\hline $\mathrm{ADCc} / \mathrm{ADCw}$ & -0.4884 & 0.1276 & -0.2612 & 0.5039 \\
\hline \multicolumn{5}{|c|}{$\begin{array}{l}\text { The rows are expressed as a ratio of the MR parameters } \\
\text { during compression (c-index) and without compression } \\
\text { (w-index). }\end{array}$} \\
\hline
\end{tabular}

Calculated MR parameters in pre- and during compression states are summarized in Table 1. The summary of Pearson correlation coefficients between MR and biomechanical parameters can be found in Table 2. The example of MR appearance of the articular cartilage sample is depicted in Fig.3.

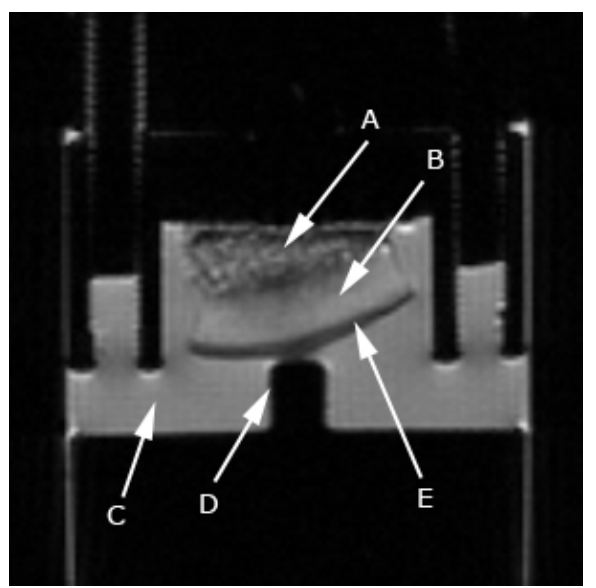

Fig.3 Cartilage specimen in micro-imaging system (IR, TI $=60 \mathrm{~ms}$, $\mathrm{TR}=4 \mathrm{~s}, \mathrm{TE}=15 \mathrm{~ms}$ ): $\mathrm{A}$ - bone tissue, $\mathrm{B}$ - deep zone of cartilage, $\mathrm{C}$ PBS + Gd-DTPA ${ }^{2-}$ solution, D - compressive piston, E - superficial zone of cartilage.

\section{DISCUSSION}

It is generally known that the response of cartilage to compressive load is not uniform [11]. Therefore, the ability to spatially localize the response of cartilage to compression is necessary. While interpreting data in comparison with in-vivo conditions there are two important factors affecting the resulting deformation: first one is the dynamics of loading and second one is the distribution of loading. Dynamics of loading strongly affects the load displacement relationship. $\mathrm{T}_{2}$ values decreased after compression by $10 \%$. These observations agree well with published results and support the hypothesis that cartilage compression results in greater anisotropy of superficial collagen fibers, because region of interest was defined very close to piston head, i.e. mostly in superficial part of cartilage. Eckstein et al. demonstrated a 5 to $6 \%$ decrease in patellar cartilage volume after compressive loading induced by performing 50 deep knee bends [12]. Besides, tissue consolidation and the resulting decrease in water content due to efflux of water as a possible factor responsible for the observed change in cartilage $\mathrm{T}_{2}$ prior ex vivo studies designed for the evaluation of changes in collagen fiber orientation with loaded conditions suggest that changes in fiber orientation is the dominant factor for $T_{2}$ shortening [13]. $T_{1}$ values of cartilage after penetration of Gd-DTPA ${ }^{2-}$ allow assessment of the proteoglycan (GAG) component of articular cartilage [14]. Applying compression to the cartilage sample invokes changes in water content in cartilage tissue. The amount of proteoglycans stays unaltered, but the amount of liquid part decreases. Due to squeezing the liquid content from cartilage, concentration of Gd-DTPA ${ }^{2-}$ decreases as well. In contrast to $\mathrm{T} 1 \mathrm{Gd}$, which reflects changes in chemical composition, diffusion constants may reflect the microstructural degradation 
of the cartilage matrix. ADC correlates with structural changes rather than with changes in chemical composition [15]. Compression may induce cartilage matrix impairment which leads to the decrease of ADC. Since $E q$ and $I$ reflect dynamic mechanics of cartilage, they correlate mostly with proteoglycan content $\left(\mathrm{T}_{1 \mathrm{Gd}}\right)$ and diffusivity of water molecules (ADC).

\section{CONCLUSION}

In conclusion, the compression device used in this study provides a convenient way to quantitatively evaluate loadinduced changes in human cartilage with extremely high accuracy and shows good correlation of biomechanical quantities with MR parameters.

\section{ACKNOWLEDGMENT}

Funding for this study was provided by the Austrian Science Fund (FWF) FWF-Project P-18110-B15 and Slovak Scientific Grant Agency VEGA 2/0142/08.

\section{REFERENCES}

[1] Mlynarik, V., Trattnig, S. (2000). Physicochemical properties of normal articular cartilage and its MR appearance. Investigative Radiology, 35, 589-594.

[2] Burstein, D., Bashir, A., Gray, M.L. (2000). MRI techniques in early stages of cartilage disease. Investigative Radiology, 35, 622-638.

[3] Herberhold, C., Stammberger, T., Faber, S., Putz, R., Englmeier, K.H., Reiser, M., Eckstein, F. (1998). An MR-based technique for quantifying the deformation of articular cartilage during mechanical loading in an intact cadaver joint. Magnetic Resonance in Medicine, 39, 843-850.

[4] Sah, R.L.Y., Kim, Y.J., Doong, J.Y.H., Grodzinsky, A.J., Plaas, A.H.K., Sandy, J.D. (1989). Biosynthetic response of cartilage explants to dynamic compression. Journal of Orthopaedic Research, 7, 619-636.

[5] Stammberger, T., Herberhold, C., Faber, S., Englmeier, K.H., Reiser, M., Eckstein, F. (1998). A method for quantifying time dependent changes in MR signal intensity of articular cartilage as a function of tissue deformation in intact joints. Medical Engineering \& Physics, 20, 741-749.
[6] Kaufman, J.H., Regatte, R.R., Bolinger, L., Kneeland, J.B., Reddy, R., Leigh, J.S. (1999). A novel approach to observing articular cartilage deformation in vitro via magnetic resonance imaging. Journal of Magnetic Resonance Imaging, 9, 653-662.

[7] Eckstein, F., Tieschky, M., Faber, S., Englmeier, K.H., Reiser, M. (1999). Functional analysis of articular cartilage deformation, recovery, and fluid flow following dynamic exercise in vivo. Anatomy and Embryology, 200, 419-424.

[8] Juras, V., Szomolanyi, P., Majdisova, Z., Tratttnig, S. (2008). MR-compatible compression device for in-vitro evaluation of biomechanical properties of cartilage. Journal of Biomechanical Science and Engineering, 3, 200-208.

[9] Markwardt, C.B. (2008). Non-linear least squares fitting in IDL with MPFIT. In Astronomical Data Analysis Software and Systems XVIII : Proceedings. Vol. 411. Quebec, Canada: ASP, 251-254.

[10] Hayes, W.C., Keer, L.M., Herrmann, G., Mockros, L.F. (1972). A mathematical analysis for indentation tests of articular cartilage. Journal of Biomechanics, 5, 541-51.

[11] Wong, M., Carter, D.R. (2003). Articular cartilage functional histomorphology and mechanobiology: a research perspective. Bone, 33, 1-13.

[12] Eckstein, F., Tieschky, M., Faber, S.C., Haubner, M., Kolem, H., Englmeier, K.H., Reiser, M. (1998). Effect of physical exercise on cartilage volume and thickness in vivo: MR imaging study. Radiology, 207, 243-248.

[13] Mosher, T.J., Smith, H.E., Collins, C., Liu, Y., Hancy, J., Dardzinski, B.J., Smith, M.B. (2005). Change in knee cartilage T2 at MR imaging after running: a feasibility study. Radiology, 234, 245-9.

[14] Burstein, D., Velyvis, J., Scott, K.T., Stock, K.W., Kim, Y.J., Jaramillo, D., Boutin, R.D., Gray, M.L. (2001). Protocol issues for delayed Gd(DTPA)(2-)-enhanced MRI (dGEMRIC) for clinical evaluation of articular cartilage. Magnetic Resonance in Medicine, 45, 36-41.

[15] Mlynarik, V., Sulzbacher, I., Bittsansky, M., Fuiko, R., Trattnig, S. (2003). Investigation of apparent diffusion constant as an indicator of early degenerative disease in articular cartilage. Journal of Magnetic Resonance Imaging, 17, 440-444. 